\title{
Importancia de los cobertores vegetales transformados para la sustentabilidad ganadera en el noreste del Cesar, Colombia
}

\section{The importance of the transformed vegetal blankets for cattle sustainability in the Northeast of Cesar, Colombia}

\author{
Roenes G, Gustavo ${ }^{1 *}$ Lic; Reales A, Juan² M.Sc \\ ${ }^{1}$ Universidad Popular del Cesar, Departamento de Ciencias Naturales y Educación Ambiental, \\ Valledupar, Cesar, Colombia. \\ ${ }^{2}$ Universidad Popular del Cesar. Valledupar, Cesar, Colombia.
}

\section{KEYWORDS:}

Climate change; global warming; Guacochito Cesar; landscape unit; botanical inventory; forage species.

\section{ABSTRACT}

The purpose of this research was to determine the structure of the vegetation that forms the transformed landscape units of the municipality of Guacochito Cesar, with the purpose of finding woody species with nutritional potential that could be used as food supplement for the sustenance of livestock in dry seasons. The sampling unit corresponded to 0,1 ha distributed in 10 plots (transects) of $50 \mathrm{~m}$ long by $2 \mathrm{~m}$ wide, covering the internal variability of the inventoried area, as developed in VILLAREAL (2014). Determinant results were obtained to establish that the transformed vegetal blankets adjacent to the district of Guacochito Cesar has a great potential, because in these units woody species with important forage characteristics such as Prosopis juliflora and Samanea saman are grown, which showed significant indices of value of importnace (IVI), which, joined to their agroecosystemic properties, allows to think about the planning of a program of rational use of these organisms in order to nutritionally supplement cattle and minor species in times of prolonged drought.

\section{RESUMEN}

El objetivo de esta investigación estuvo encaminada a determinar la estructura de la vegetación que conforma las unidades paisajísticas transformadas del municipio de Guacochito Cesar, con el propósito de hallar especies leñosas con potencial nutricional que bien pudieran ser usadas como suplemento alimentario para el sostenimiento de del ganado en temporadas secas. La unidad de muestreo correspondió a 0,1 ha distribuida en 10 parcelas (transectos) de $50 \mathrm{~m}$ de largo por $2 \mathrm{~m}$ de ancho, abarcando la variabilidad interna del área inventariada, según lo desarrollado en VILLAREAL (2014). Se obtuvieron resultados determinantes para establecer que los cobertores vegetales transformados adyacentes al corregimiento de Guacochito Cesar poseen un gran potencial, debido a que en estas unidades prosperan especies leñosas con características forrajeras importantes tales como Prosopis juliflora y Samanea saman, las cuales mostraron índices de valor de importancia (IVI) significativos, que sumado a sus propiedades agroecosistémicas, permiten pensar en la planificación de un programa de uso racional de estos organismos con el fin de suplementar nutricionalmente al ganado bovino y especies menores en las épocas de sequía prolongada. 


\section{INTRODUCCIÓN}

Los efecto del cambio climático, definido por OROZA (2012) como el cambio del sistema climático de la tierra, y el calentamiento global precisado por BARBOSA (2013) como el calentamiento de la tierra debido a actividades humanas, son fenómenos preocupantes que viene sufriendo el Planeta en las últimas décadas, tal como lo indican entre otros, DNP (2015); CEPAL (2013), ABUD y GUEVARA (2013), situación que ha generado fuertes sequias en la región Caribe de Colombia principalmente en el departamento del Cesar, ocasionado la muerte de cientos de cabezas de ganado bovino y especies menores sin que se encuentre a corto o mediano plazo una solución a este problema.

La Federación Colombiana de Ganaderos (FEDEGAN, 2017) ha revelado información en el último lustro sobre los casos sistemáticos de muerte de ganado por el grave efecto de las sequias. En este contexto, el Cesar ha sido uno de los departamentos del País más afectado por esta problemática, en la cual, según el Fondo Nacional del Ganado (FNG), los fuertes estaciones secas han ocasionado que cada vez se presenten más casos de muerte masiva de animales. Información suministrada por esta entidad, reconoce que en el año 2015 las muertes ocasionadas por este fenómeno climático ascendieron a 2.782 cabezas, situación que ha causado reiteradamente manifestaciones de descontento de los productores al no encontrar respuesta a esta crisis.

De acuerdo a información obtenida a través del Departamento de Planeación Nacional (DPN et al., 2015), el comportamiento del cambio climático, en general, tendrá una incidencia negativa para el sector ganadero del país, correspondiente a la estimación de los cambios en la productividad de las pasturas bajo diferentes escenarios de cambio climático propuestos por el Panel Intergubernamental de Cambio Climático (IPCC), mismos adoptados por el Instituto de Estudios Ambientales del País (IDEAM), también usados por el gobierno nacional para realizar sus proyecciones. Para el departamento del Cesar, las modelaciones se realizaron con información climática de dos estaciones meteorológicas del IDEAM, pronosticando que en todos los escenarios la tendencia es a la disminución en el rendimiento de las pasturas durante todo el siglo. De esta manera, dos de los escenarios modelados predijeron la pérdida de hasta 1,7 toneladas por ha, mientras que un tercero mostró una tendencia a la pérdida de hasta 2,5 toneladas de materia seca por ha, a finales de este siglo.

Es evidente que la temperatura de la región de la cuenca del río Cesar ha tenido una tendencia a incrementarse paulatinamente en las últimas décadas. Según HURTADO (2016), en la década del año 1971 a
1980 la temperatura promedio anual estuvo en $33^{\circ} \mathrm{C}$; entre el año 1981 a 1990 se marcó una temperatura promedio de $33,46{ }^{\circ} \mathrm{C}$; en los años de 1991 al 2000 la temperatura ascendió a $34,09^{\circ} \mathrm{C}$ y en la década del 2001 al 2010, se registró una temperatura promedio de $33,92^{\circ} \mathrm{C}$. Estudios realizados por el IDEAM et al. (2015), sustentan de manera pormenorizada que hasta el año 2100 la temperatura promedio de Colombia, que hoy está en 22 grados, subiría dos grados centígrados adicionales, ese aumento sería gradual, ya que de aquí al 2040 está contemplado un ascenso de 0,9 grados y hasta 2070 ese incremento llegaría a los 1,6 grados promedio. De acuerdo a esto, la región Caribe será más seca porque alli lloverá casi un $15 \%$ menos. Las lluvias bajarán en un $27 \%$ en el territorio nacional, reducción que será entre un 10 y un $30 \%$, incluyendo a Amazonas, Vaupés, sur de Caquetá, Bolívar, Sucre, norte del Cesar, La Guajira, Atlántico, San Andrés y Providencia y Magdalena. Igualmente en Meta y Casanare (Orinoquia).

Bajo estas condiciones negativas, para las cuales no se avizora una solución pronta, se hace necesario empezar a producir iniciativas locales que resguarden y recuperen la actividad ganadera de la región norte del Cesar, mediante el desarrollo de estrategias sustentables que eviten la pérdida del ganado por inanición, sobre todo en las épocas de sequias extensas.

El uso de especies vegetales nativas con potencial forrajero resistentes al aumento de las temperaturas y a sequías prolongadas, como complemento nutricional para el ganado bovino y especies menores, es un alternativa viable que puede marcar un cambio sustancial para sostener la actividad pecuaria en zonas de climatología variante afectadas por el cambio climático, cual es el caso de la región nororiental del departamento del Cesar (CHUNCHO, 2011).

El corregimiento de Guacochito se ubica en la región nororiental del departamento del Cesar en límites con el departamento de La Guajira colombiana. En su mayoría, sus moradores son de origen Afro, siendo este un elemento fundamental desde el punto de vista político, pues cuentan con la autonomía necesaria que les permite tomar decisiones frente al futuro social y económico de sus habitantes, lo cual contrasta con las deficiencias y necesidades que sufre la mayor parte de la población al no contar con servicios públicos, centros educativos, espacios recreativos, puestos de salud o núcleos comerciales que faciliten una mejor calidad de vida. Particularmente la población se dedica a la producción agrícola y la crianza de ganado bovino y especies menores en minifundios que colindan con hatos ganaderos latifundistas de propiedad foránea, es decir, que pertenecen a personas ajenas a la población nativa (HERRERA et al., 2017). 
La comunidad cuenta con zonas comunes de pastoreo y también con parcelas privadas de área reducida que comprenden sistemas silvopastoriles, en la cual los elementos naturales remanentes del bosque seco tropical se asocian con especies herbáceas de porte deprimido y pastos que se usan, como habría de esperarse, para la alimentación de vacas, chivos, cerdos y aves de corral. Lastimosamente no existe un plan generalizado de aprovechamiento sostenible de las especies leñosas nativas para mejoramiento de la nutrición del ganado o para soportar las temporadas de sequía cuando las pasturas desaparecen, lo cual es coherente con los pocos estudios realizados en esta región del departamento del Cesar relacionados con determinar la estructura de la cobertura vegetal y con ello, cuantificar y valorar las especies arborescentes que poseen un potencial para ser aprovechadas en la alimentación animal.

Teniendo en cuenta los aspectos antes citados, el objetivo principal de este trabajo se centró en la caracterización de la vegetación leñosa asociadas a las unidades paisajísticas (UP) agroecosistémicas o a las zonas sucesionales aledañas al corregimiento de Guacochito Cesar, con el fin de identificar especies vegetales con potencial nutricional valioso que puedan ser usadas para contribuir al sostenimiento alimentario del ganado bovino y especies menores en las temporadas de verano intenso.

\section{MATERIALES Y MÉTODOS}

Área de estudio. según el PLAN DE DESARROLLO DEPARTAMENTAL (2012-2015), el departamento del Cesar cuenta con una superficie de $22.925 \mathrm{~km}^{2}$, lo que representa el 2,0\% del total nacional. En su territorio se encuentra una gran diversidad de pisos térmicos y climas, por lo tanto las temperaturas fluctúan entre $4^{\circ} \mathrm{C}$ y $38^{\circ} \mathrm{C}$. El Departamento posee 25 municipios, con una población total de 979.054 habitantes, de la cual el $73,22 \%$ es urbana y el $26,78 \%$ es rural.

De acuerdo a datos obtenidos del PLAN DE DESARROLLO DE VALLEDUPAR (2012-2015), el municipio de Valledupar está ubicado en los $10^{\circ} 29$ > de latitud Norte y $73^{\circ} 15$ > de longitud Oeste; es el más extenso del departamento $\left(4.264,8 \mathrm{~km}^{2}\right)$ ocupando el $18,8 \%$ de su territorio total. El área rural corresponde al $99,14 \%$ del Municipio y la cabecera ocupa cerca de 27 $\mathrm{km}^{2}$. El Municipio se encuentra ubicado en el conjunto geográfico de la Sierra Nevada de Santa Marta, la cual representa uno de los paisajes geográficos de mayor variedad y climas por encontrarse a diferentes altitudes que van desde el ecuatorial o cálido, subandino o templado, andino o frío hasta el páramo, con temperaturas que van de 0 hasta $32^{\circ} \mathrm{C}$. El área rural del Municipio se encuentra compuesta por 24 corregimientos distribuidos en seis zonas. La zona nororiental se corresponde geográficamente con los corregimientos de Patillal, Badillo, El Alto de la Vuelta, Guacoche, Las Raíces, Los Corazones, La Vega Arriba y Guacochito.

La investigación tuvo como epicentro el corregimiento de Guachito, el cual se ubica geográficamente a $10^{\circ} 30^{\prime} 56^{\prime \prime}$ de latitud Norte y $73^{\circ} 08^{\prime} 59^{\prime \prime}$ de longitud Oeste, ver panorámica satelital (Fig. 1). Posee una extensión total de 2.147 ha y una altitud de $165 \mathrm{~m}$ sobre el nivel del mar; limita al norte con el corregimiento del Alto de la Vuelta, al sur con los corregimientos de Guacoche, Badillo y Urumita, y al oeste con los corregimientos de Las Raíces y Los Corazones. Su hidrografía está representada por los Ríos Cesar y Badillo, siendo su temperatura media característica de $32^{\circ} \mathrm{C}$ (PLAN DE DESARROLLO DE VALLEDUPAR, 2012-2015).
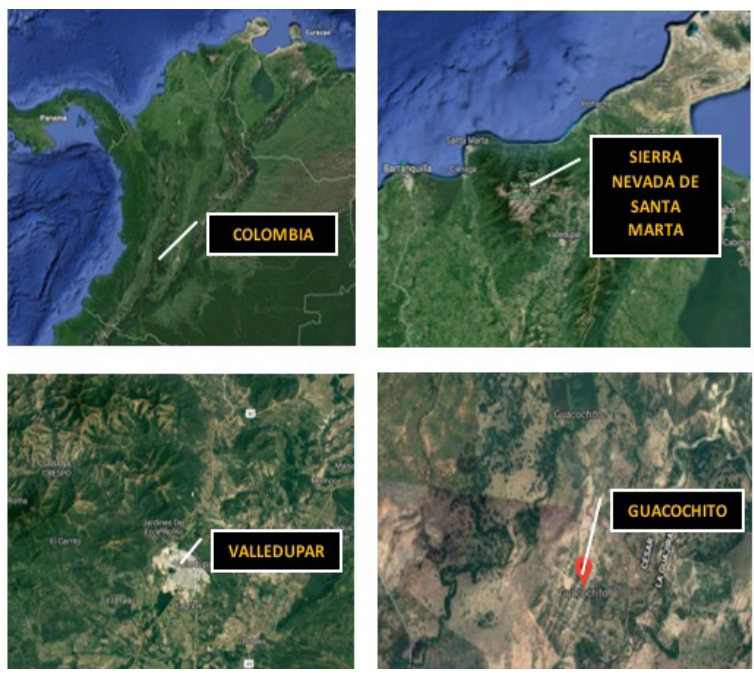

Figura 1. Panorámica satelital del Corregimiento de Guacochito Cesar. Fuente: Google-Maps, 2017.

Los orígenes históricos del corregimiento de Guacochito se remontan a finales del siglo XIX, cuando viajeros descendientes de esclavos transitaban desde La Guajira hacia el interior del Caribe por los callejones de origen colonial existentes en el norte de Valledupar. La abundancia de agua, la fertilidad del suelo y la extensión de tierras libres de aquellos tiempos, fueron convirtiendo los lugares de paso en asentamiento permanentes. Así, en las planicies de la cuenca del río Cesar se conformaron tres caseríos que son los puntos de referencia en la historia del poblamiento: Paredones, Palmarito y Guacochito. Desde entonces, el Corregimiento ha tenido su desarrollo en las sabanas ribereñas del Río Cesar, el cual ha sido una fuente vital para su poblamiento, al igual que las formaciones de bosque seco tropical aprovechado comunitariamente desde siempre por sus habitantes (HERRERA et al., 2017). 
El Corregimiento fue seleccionado para el desarrollo de la investigación, teniendo en cuenta las necesidades insatisfechas de la población y también por ser una de las localidades del departamento del Cesar más afectadas por el impacto del cambio climático y el calentamiento global, sumado a los procesos de degradación de suelos, desertización y pérdida de sus fuentes hídricas. Estos factores han sido críticos en la dinámica cultural y social de sus moradores, teniendo en cuenta que la escasez de lluvias en los últimos años ha generado un detrimento de las actividades económicas relacionadas principalmente con la cría de ganado y especies menores (GUTIÉRREZ, 2016).

El punto de partida de la investigación estuvo marcado por las entrevistas sostenidas con los moradores en el marco de diferentes escenarios académicos y sociales realizados con esta comunidad, en la cual se focalizaron diferentes tensiones experimentadas en diferentes aspectos de su cotidianidad, en especial, lo relativo a las dificultades causadas por las temporadas de sequía prolongada. Las discusiones generadas al respecto, determinaron la necesidad de encontrar opciones agronómicas que permitieran la sostenibilidad de la ganadería en esos periodos largos de verano.

Una vez detectado el problema objeto de investigación, se promovieron jornadas de participación comunitaria, sobre todo con la población más afectada, para establecer diferentes alternativas de solución. La respuesta más viable, consistió en hacer una caracterización de la riqueza florística de la zona de influencia, teniendo en cuenta que este tipo de estudios (inventarios) describen la estructura y función de la vegetación para su aplicación en el uso y manejo de la misma (CAMPO, 2014), en especial la determinación de la estructura poblacional de los cobertores vegetales transformados con el fin de identificar especies leñosas con potencial nutricional que bien pudieran ser aprovechadas como suplemento nutricional para el ganado bovino y especies menores en las temporadas estivales. Este estudio se justificó por demás, dado el poco conocimiento de la población sobre el uso racional de especies leñosas para la alimentación de los animales.

En esta medida, se pudo establecer que los minifundistas oriundos del corregimiento, no hacen uso significativo de las especies leñosas nativas para la alimentación del ganado, excepto por el patrón natural que siguen los animales de ramajear y comer los frutos principalmente de especies leguminosas, mismas que son usadas por los propietarios para propósitos diferentes como por ejemplo, fuente de madera y combustible.

En este sentido, el análisis de la abundancia y el valor nutricional de especies nativas de la zona puede incidir finalmente en coadyuvar el fortalecimiento de las condiciones de vida de los habitantes de este corregimiento, gracias al aporte nutricional que algunas de estas especies forestales puede aportar para el mantenimiento del ganado bovino y especies menores, sobre todo en temporadas de sequías prolongadas, cuando los pastos desaparecen.

Unidad paisajística (UP): las etapas iniciales de planeación y el trabajo de campo se desarrollaron a partir del análisis de sensores remotos disponibles en la Web, tal es el caso de Google Erth, Google Maps y otros servidores que operan en línea, complementándose la información con el reconocimiento del paisaje mediante exploraciones de campo, entrevistas a los moradores e investigaciones bibliográficas (LEYVA, 2011; VILLAREAL et al., 2014).

La unidad de muestreo aplicada aquí, fue la unidad UP, la cual se define como el área discreta de la superficie del terreno, apta para ser muestreada y cartografiada a cualquier nivel de resolución, (VILLAREAL et al., 2014). En la caracterización de las unidades paisajísticas (UP) se usaron sensores remotos digitales para definir los atributos físicos de cada UP, mediante el análisis de la diferenciación de patrones de coloración con el fin de representar en papel la unidad de mapeo (UM) general. Una vez realizado esto, se procedió a la verificación en campo del mapeo inicial, y se confirmó la UP objeto de ser muestreada, teniendo en cuenta su mayor biodiversidad, representatividad y distintividad.

Inventario botánico: el inventario botánico fue realizado en un sector ubicado entre el límite sureste del poblado y la margen derecha del río Cesar. Zona que predominantemente se encuentra estructurada por especies arborescentes (árboles y arbustos) en diferentes estados de desarrollo en asocio con vegetación de bajo porte principalmente malezas y pastos de diferentes especies, lo cual representa un estado transformado de la cobertura vegetal (Figura 2).

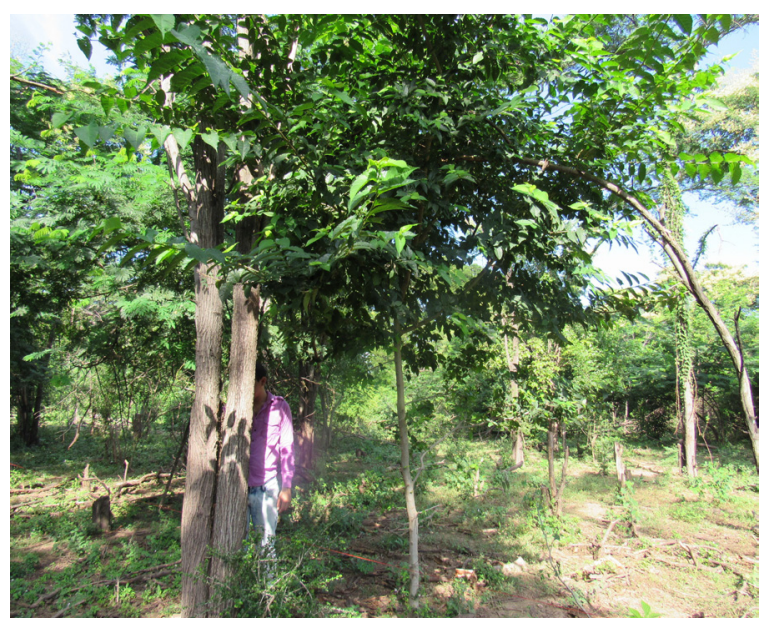

Figura 2. Características de la zona inventariada. Fuente propia, 2016 
La unidad de muestreo correspondió a 0,1 ha distribuida en 10 transectos de $50 \mathrm{~m}$ de largo por $2 \mathrm{~m}$ de ancho, teniendo presente que no se superpusieran y estuvieran separados por una distancia de hasta 20 m (Tabla 1), abarcando la variabilidad interna de la UP inventariada, con un esfuerzo de muestreo para determinación de la riqueza de plantas leñosas cuya circunferencia a la altura del pecho (CAP) fuera mayor o igual a $7 \mathrm{~cm}$ (VILLAREAL ,2014).

Tabla 1. Localización geográfica de los transectos correspondientes a los muestreos realizados.

\begin{tabular}{ccccc} 
Transecto & \multicolumn{2}{c}{$\begin{array}{c}\text { Georeferenciación } \\
\text { Lat }\end{array}$} & $\begin{array}{c}\text { Lon } \\
\text { Altitud } \\
(\mathbf{m})\end{array}$ & $\begin{array}{c}\text { Fecha } \\
\text { (DD/MM/AA) }\end{array}$ \\
1 & $10^{\circ} 30.554^{\prime}$ & $73^{\circ} 8.861^{\prime}$ & 148 & $26 / 11 / 16$ \\
2 & $10^{\circ} 30.557^{\prime}$ & $73^{\circ} 8.824^{\prime}$ & 159 & $01 / 12 / 16$ \\
3 & $10^{\circ} 30.580^{\prime}$ & $73^{\circ} 08.834^{\prime}$ & 138 & $01 / 12 / 16$ \\
4 & $10^{\circ} 30.591^{\prime}$ & $73^{\circ} 08.840^{\prime}$ & 147 & $01 / 12 / 16$ \\
5 & $10^{\circ} 30.600^{\prime}$ & $73^{\circ} 08.847^{\prime}$ & 143 & $01 / 12 / 16$ \\
6 & $10^{\circ} 30.611^{\prime}$ & $73^{\circ} 08.852^{\prime}$ & 140 & $01 / 12 / 16$ \\
7 & $10^{\circ} 30.622^{\prime}$ & $73^{\circ} 08.859^{\prime}$ & 149 & $01 / 12 / 16$ \\
8 & $10^{\circ} 30.636^{\prime}$ & $73^{\circ} 08.865^{\prime}$ & 139 & $01 / 12 / 16$ \\
9 & $10^{\circ} 30.651^{\prime}$ & $73^{\circ} 8.874^{\prime}$ & 141 & $01 / 12 / 16$ \\
10 & $10^{\circ} 30.665^{\prime}$ & $73^{\circ} 08.895^{\prime}$ & 140 & $01 / 12 / 16$
\end{tabular}

Una vez realizado el inventario se procesó la información en hojas de cálculo de Excel con el fin de establecer los rasgos estructurales de la (UP) muestreada, con el fin de establecer el índice del valor de importancia (IVI), el cual se obtuvo mediante la sumatoria de la frecuencia relativa, la densidad relativa y la dominancia relativa de cada morfoespecie, CAMPO (2014). Al final el IVI indicaría la riqueza de especies leñosas con potencial para ser usadas en la alimentación del ganado bovino y especies menores durante las temporadas largas de sequía.

\section{RESULTADOS}

Una vez procesada la información de campo, consistente en el inventario botánico realizado con el fin de obtener los rasgos estructurales de la (UP) inventariada, se encontró una densidad total de 477 individuos clasificados en 22 morfoespecies diferentes, registrándose una cobertura (área basal total) de 64.416 $\mathrm{cm}^{2}$, tal como se muestra en la Tabla 2.

El parámetro más importantes que definió el estudio, fue la determinación del índice del (IVI) de las morfoespecies encontradas: es importante explicar que

Tabla 2. Parámetros estructurales de los muestreos en 0,1 ha.

\begin{tabular}{|c|c|c|c|c|c|c|c|c|c|c|c|c|}
\hline M & Familia & Género & Especie & Nombre vulgar & D & DR & TA & $\mathbf{F}$ & FR & $A B$ & ABR & IVI \\
\hline 1 & Fabaceae & Samanea & saman & Algarrobillo & 40 & 0,008 & 4 & 0,4 & 0,0513 & 38400,4 & 0,5961 & 0,6558 \\
\hline 2 & Fabaceae & Acacia & farnesiana & Aromo & 50 & 0,010 & 2 & 0,2 & 0,0256 & 182,3 & 0,0028 & 0,0390 \\
\hline 3 & Fabaceae & Haematoxylum & brasiletto & Brasil & 140 & 0,029 & 3 & 0,3 & 0,0385 & 264,6 & 0,0041 & 0,0719 \\
\hline 4 & Capparaceae & Quadrella & odoratissima & Buchepavo & 10 & 0,002 & 1 & 0,1 & 0,0128 & 2283,1 & 0,0354 & 0,0504 \\
\hline 5 & Bignoniaceae & Tabebuia & chrysantha & Cañahuate & 180 & 0,038 & 5 & 0,5 & 0,0641 & 375,7 & 0,0058 & 0,1077 \\
\hline 6 & Fabaceae & Pithecellobium & forfex & Cariotico & 70 & 0,015 & 3 & 0,3 & 0,0385 & 157,4 & 0,0024 & 0,0556 \\
\hline 7 & Zygophyllaceae & Bulnesia & arbórea & Carrapo & 20 & 0,004 & 2 & 0,2 & 0,0256 & 313,2 & 0,0049 & 0,0347 \\
\hline 8 & Rubiaceae & Randia & $s p$ & & 320 & 0,067 & 5 & 0,5 & 0,0641 & 323,1 & 050 & 0,1362 \\
\hline 9 & Caesalpiniaceae & Caesalpinia & ebano & Ébano & 20 & 0,004 & 1 & 0,1 & 0,0128 & 481,7 & 0,0075 & 0,0245 \\
\hline 10 & Fabaceae & Acacia & polyphylla & Espinito & 200 & 0,042 & 7 & 0,7 & 0,0897 & 613,3 & 0,0095 & 0,1412 \\
\hline 11 & Fabaceae & Albizia & niopoides & Guacamayo & 10 & 0,002 & 1 & 0,1 & 0,0128 & 102,4 & 0,0016 & 0,0165 \\
\hline 12 & Apocynaceae & Thevetia & ahouai & Lechoncito & 780 & 0,164 & 7 & 0,7 & 0,0897 & 1002,8 & 0,0156 & 0,2688 \\
\hline 13 & & & & & 40 & 0,008 & 1 & 0,1 & & & & \\
\hline 14 & Malpighiacea & Malpighia & punicifolia & Mácara & 10 & 0,002 & 1 & 0,1 & 0,0128 & 6,4 & 0,0001 & 0,0150 \\
\hline 15 & Rhamnaceae & Ziziphus & ziziphus & Manzanillo & 20 & 0,004 & 2 & 0,2 & 0,0256 & 135,4 & 0,0021 & 0,0319 \\
\hline 16 & Fabaceae & Senna & atomaria & Mapurito & 90 & 0,019 & 5 & 0,5 & 0,0641 & 632,8 & 0,0098 & 0,0928 \\
\hline 17 & Moraceae & Chlorophora & tinctoria & Mora & 120 & 0,025 & 4 & 0,4 & 0,0513 & 384,8 & 0,0060 & 0,0824 \\
\hline 18 & Fabaceae & Acacia & riparia & Mulato & 250 & 0,052 & 7 & 0,7 & 0,0897 & 3230,8 & 0,0502 & 0,1923 \\
\hline 19 & Fabaceae & Enterolobium & cyclocarpum & Orejo & 10 & 0,002 & 1 & 0,1 & 0,0128 & 3999,4 & 0,0621 & 0,0770 \\
\hline 20 & Burseraceae & Bursera & simaruba & Siete cueros & 20 & 0,004 & 2 & 0,2 & 0,0256 & 237,4 & 0,0037 & 0,0335 \\
\hline 21 & Capparidaceae & Crataeva & tapia & Toco & 170 & 0,036 & 4 & 0,4 & 0,0513 & 820,5 & 0,0127 & 0,0997 \\
\hline 22 & Fabaceae & Prosopis & juliflora & Trupillo & 2200 & 0,461 & 10 & 1 & 0,1282 & 10324,4 & 0,1603 & 0,7497 \\
\hline \multicolumn{5}{|c|}{ TOTAL } & 4770 & 1,000 & & 7,8 & 1,0000 & 64416,1 & 1,0000 & 3,0000 \\
\hline
\end{tabular}


la dominancia relativa, en el caso de esta investigación, se refiere a la cobertura relativa indicada en la Tabla 2 como área basal relativa, factor necesario para poder estimar cuáles de las especies encontradas, concomitantemente a su valor nutricional, cumplían con el requisito significativo de estar bien distribuida espacialmente y presentar una magnitud volumétrica importante para los fines establecidos. Los valores del IVI (Fig. 3), predijeron un patrón bien marcado de dos (2) morfoespecies aptas relacionadas con el área de estudio: Prosopis juliflora (IVI=0,75) y Samanea saman (IVI=0,66).

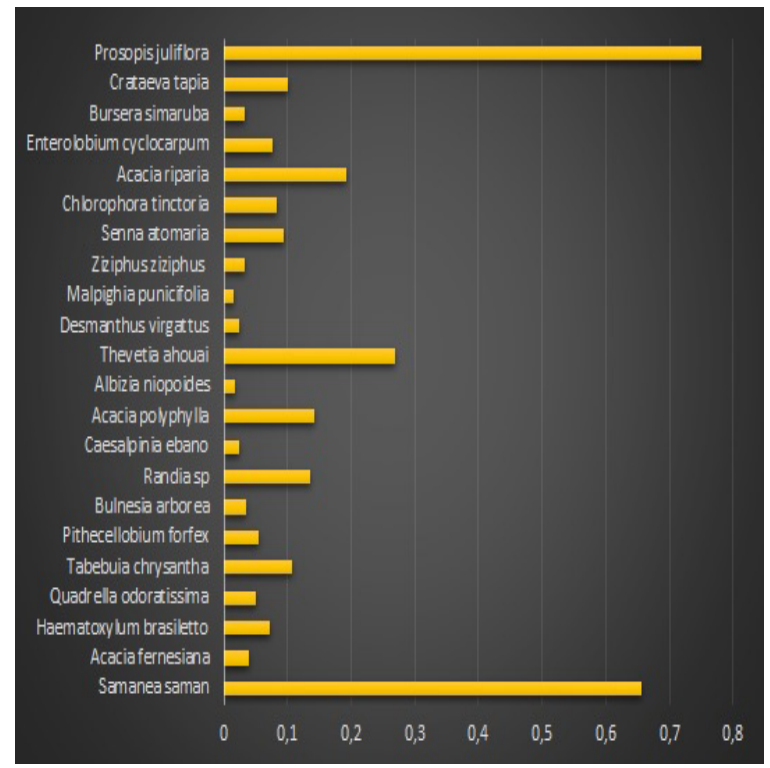

Figura 3. Índice del Valor de Importancia (IVI) de las morfoespecies halladas.

\section{RESULTADOS Y DISCUSIÓN}

Las observaciones y análisis realizados en el desarrollo de la investigación permitieron constatar que la región de Guacochito Cesar es una zona que por sus características geográficas, altitud, su climatología y condiciones edáficas, conserva vestigios de una vegetación nativa de bosque tropical seco (BST), tal como lo describe ACEVEDO (2016). Factores orográficos han sido decisivos para el florecimiento de este tipo de vegetación en la zona, por ejemplo la situación geográfica de la serranía de Perijá la cual se ubica en el costado oriental del departamento del Cesar. Esta elevación se caracteriza por poseer una vegetación de selva húmeda en el flanco venezolano contra el cual chocan los vientos alisios; en la cara colombiana es característico encontrar una vegetación de ambiente más seco, quedando fragmentos de bosque seco tropical (BST) que en los años 50 cubría buena parte del piedemonte así como hondonadas, planicies, arroyos y afluentes del Río Cesar (PIZANO y GARCÍA, 2014). Evidentemente, esta región ha tenido un gran impacto antropocéntrico relacionado con el uso actual del suelo, consistente en la explotación ganadera y la agricultura. Se observó que la zona urbana del corregimiento se encuentra bordeada por hatos ganaderos en su mayoría no pertenecientes a los moradores del corregimiento, excepto pequeños predios que se encajan entre los límites del pueblo y los cercados que delimitan las grandes explotaciones. Desde esta perspectiva, se lograron diferenciar dos estados agroecosistémicos, FARFÁN (2014), los dedicados a las grandes y medianas explotaciones ganaderas, consistentes en potreros con vegetación casi exclusiva de gramíneas usadas para alimento de ganado vacuno, y pequeñas áreas de vegetación transformada muy próximas al poblado que conservan un patrón de vegetación natural arborescente asociada con pastos y especies de plantas de porte deprimido (Figura 4).
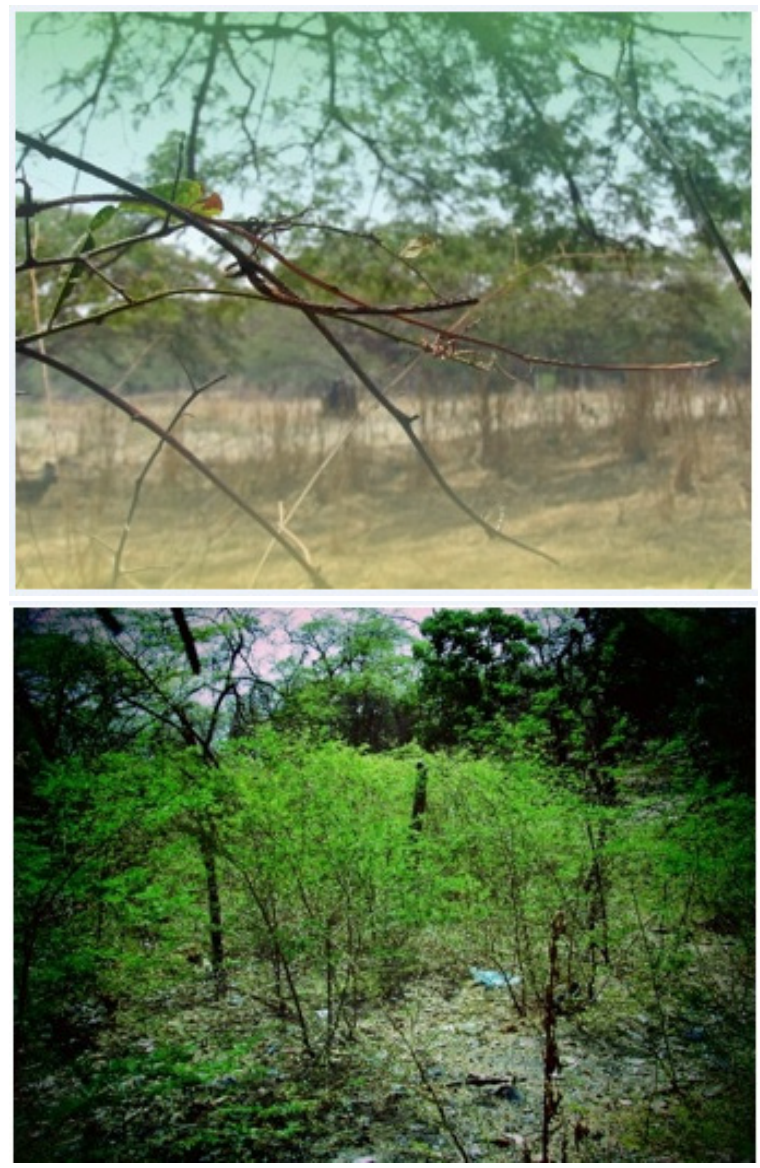

Figura 4. Estados agroecosistémicos típicos en la población de Guacochito Cesar. La imagen de la izquierda corresponde a las áreas de pastoreo usadas para explotación ganadera, y la imagen de la derecha muestra pequeñas zonas muy próximas al poblado compuestas por especies arborescentes naturales asociadas con pastos. Fuente propia, 2016. 
Las dos morfoespecies más significativas halladas en las unidades transformadas del Corregimineto $(P$. juliflora y $S$. saman) constituyen una base significativa para uso alimentario del ganado, llamando la atención que poseen características comunes como por ejemplo, situarse taxonómicamente en el grupo botánico de las leguminosas (Fabaceae). Los usos que se le han atribuido a esta familia son cuantiosos, sobre todo desde el punto de vista económico, tomado en cuenta que esta familia botánica sólo se sitúa por detrás de las Gramíneas en importancia económica a nivel mundial. Algunas especies de leguminosas se caracterizan por la calidad de la madera, mientras otras son forrajeras (FORERO y ROMERO, 2005; SARMIENTO, 2012).

Desde esta perspectiva, son valiosos los estudios que demuestran las bondades nutricionales de $P$. juliflora (Trupillo), sobre todo para zonas de climatología tropical con escases de lluvias. Esta especie se caracteriza por sus múltiples usos, teniendo en cuenta que sus frutos contienen un alto contenido de carbohidratos y proteínas. La pulpa de las vainas son dulces y las semillas contienen entre 34 y $39 \%$ de proteínas, y entre 7 y $8 \%$ de aceites. Como forraje, las vainas concentran cerca de 8 a $10 \%$ de proteína bruta y una digestibilidad por encima de $74 \%$. En cuanto al follaje la cantidad de proteína es del $18 \%$ y la digestibilidad llega a un $59 \%$, aunque es de baja palatabilidad y posee taninos. No solo los beneficios de esta especie se asocian a la nutrición, también se ha demostrado su contribución efectiva en los procesos de nitrificación y el uso racional del agua sin detrimento del follaje en temporadas secas (ARNERO, 2015). Por otro lado, S. saman (Algarrobillo), especie no tan densa como la anterior pero no menos importante que aquella, se sitúa entre las especies botánicas de sur América con mayor potencial, siendo su uso maderable el más provechoso en esta parte de Colombia.

En el marco de un estudio naturalístico realizado con fines de elaborar el plan de manejo ambiental del Santuario de Vida Silvestre "Los Besotes" ubicado a pocos kilómetros de Valledupar, se refiere a esta especie como un árbol de hasta $20 \mathrm{~m}$ de altura, de copa ancha y diámetro del tronco de hasta $1,5 \mathrm{~m}$ con múltiples usos, que crece en formaciones vegetales de bosque seco tropical, con un carácter especial: de cerrar sus hojas cuando amenaza lluvia, de ahí otro de sus nombres "Árbol de lluvia" (BARBOSA et al., 2008).

Análisis bromatológicos realizados (DELGADO et al., 2014), establecen que $S$. saman es una alternativa con gran potencial para alimento del ganado, lo cual se corresponde con los análisis comparativos de la composición química del follaje de la especie producto de investigaciones realizadas en Colombia, Venezuela y Cuba. Según estos análisis, los valores reportados para proteína cruda en promedio estuvieron por encima del $20 \%$, similar a lo informado por el mismo estudio para otras leguminosas forrajeras tropicales; la fracción fibrosa del follaje mostró valores de fibra neutro detergente (FND) cercanos al $46,3 \%$ y para fibra ácido detergente arrojó valores del $33,2 \%$. En cuanto a sustancias grasas también encontrada en follaje e importantes por su valor energético, se obtuvieron valores de $4,4 \%$ superior a un $3,3 \%$ informado en estudios comparativos sobre otras especies relacionadas.

Los estudios realizados por JUÁREZ (2004) citados en el trabajo aludido anteriormente, determinaron valores de lignina entre 6 y $14 \%$, lo cual se explica en la misma investigación mediante el carácter funcional que la especie manifiesta de intensificar la lignificación de las paredes celulares del follaje entre los meses de mayo a octubre, lo cual propone un elemento de estudio más específico teniendo en cuenta que el incremento de la lignina tiende a limitar la palatabilidad natural del material vegetal.

SOLANO (2013), hace referencia de los análisis realizados por RONCALLO (1997) a los frutos de $S$. saman, en la cual se expresan favorablemente los resultados obtenidos por cuanto el valor porcentual de proteína encontrada fue de $29,3 \%$, FDA $18,7 \%$, FDN $22,9 \%$ y carbohidratos solubles $40,9 \%$, concluyéndose que los frutos de la especie contienen un mayor porcentaje de proteínas y carbohidratos que el follaje, y un menor volumen de pared celular lo cual facilita su digestibilidad y por ende incrementa el valor nutricional.

Las investigaciones que se han realizado sobre estas dos especies ponen de manifiesto sus múltiples bondades, no solamente refiriéndose a su valor pecuario, también poniendo en evidencia su gran valor ecológico asociado a la capacidad de nitrificación del suelo por ser plantas que tienen la capacidad de asociarse simbióticamente con bacterias nitrificantes, lo cual es un aspecto edáfico que no se puede perder de vista (PAREDES, 2013). Por ejemplo, S. samam es una excelente opción para el mejoramiento de la pastura porque su capacidad de fijación de nitrógeno mejora la calidad nutricional de las especies de gramíneas que crecen cerca y debajo de los árboles; el humus o mantillo, producto de la descomposición de la materia orgánica del sus hojas frescas y la hojarasca que se acumulan debajo de los árboles, constituyen una verdadera fuente de nitrógeno para enriquecer el suelo, no obstante es poco frecuente la técnica de cortar sus hojas y ramas para tal efecto, pues la tasa de renovación foliar de $S$. samam es relativamente lenta en comparación con otras especies nitrógeno fijadoras como por ejemplo Gliricidia sepium, Calliandra calothyrsus y Leucaena leucocephala (STAPLES y ELEVITCH, 2006). 
Por otro lado, el volumen del dosel arbóreo, cual es el caso S. saman, proporciona sombrío necesario a los animales durante las horas más calurosas del día y en temporadas secas cuando el aumento de la temperatura es más acentuado. Relacionado con esto, la cobertura vegetal de estas especies es un elemento que interfiere con la radiación solar directa y por lo tanto con la tasa de transpiración y evaporación de las especies vegetales deprimidas que prosperan bajo sus ramas y el suelo que se encuentran por debajo de ellas. El florecimiento que finaliza cuando empieza la temporada de sequía permite la producción de frutos durante la estacionalidad seca, lo cual es un excelente indicador de la importancia de estas especies para contrarrestar la inanición de los animales cuando las pasturas desaparecen. Para el caso, el uso de estas especies vegetales mediante aplicaciones agrotécnicas racionales, muy bien podrían coadyuvar el mantenimiento del ganado bovino y especies menores en zonas golpeadas por el fenómeno de cambio climático y el calentamiento global que viene sufriendo el planeta, cual es el caso de la región de Guacochito Cesar (DELGADO, 2014).

En conclusión las áreas aledañas al poblado de Guacochito Cesar que se han mantenido arborizadas con vegetación remanente de bosque seco tropical, albergan especies leñosas principalmente de plantas leguminosas con potencial nutricional que bien podrían ser usadas racionalmente para suplementar la alimentación del ganado bovino y especies menores en las temporadas de sequía prolongada.

El análisis de la estructura de la cobertura vegetal relacionada con las unidades transformadas, indicaron la prevalencia de especies con características forrajeras tales como P. juliflora (Trupillo) y S. Saman (Algarrobillo).
El estudio logró establecer que $P$. juliflora (Trupillo) es una especie muy común en la zona, encontrándose en todos los transectos muestreados, alcanzando de esta manera un (IVI) prometedor, que junto con $S$. saman (Algarrobillo), las convierten en especies de fácil consecución y uso para los fines indicados.

No obstante lo anterior, los acercamientos sostenidos al interior de la comunidad de Guacochito, evidenciaron que los propietarios o usuarios de las áreas relacionadas con cobertores vegetales transformados, poco son dados a usar estas especies leñosas para la alimentación de su ganado de manera técnica y planificada.

\section{Agradecimientos.}

A la Vicerrectoría de Extensión e Investigación de la Universidad Popular del Cesar por su apoyo institucional a los procesos académicos e investigativos. A la Pontificia Universidad Javeriana en el marco del proyecto "Trayectorias históricas y escenarios prospectivos de las reformas de tenencia colectiva de la tierra en áreas forestales comunitarias en Colombia", financiado por el Centro Internacional de Investigación Forestal (CIFOR), por invitarnos a participar de las mesas de trabajo en los años 2016 y 2017 conformadas con los consejos comunitarios de territorios Afro descendientes, principalmente aquellos circunscritos políticamente al municipio de Valledupar, y con ello permitirnos conocer la problemática y dinámica de la tenencia de la tierra de estas comunidades. Así mismo, al Consejo Comunitario del corregimiento de Guacochito Cesar, por su prestancia y colaboración en el conocimiento de su territorio y los rasgos culturales y naturales asociados al desarrollo de este trabajo.

\section{REFERENCIAS}

ABUD, M.; GUEVARA, O. 2013. Nodos Regionales de Cambio Climático, Reporte Consolidado de Línea Base Análisis de Riesgos Climáticos y Necesidades de Adaptación Climática. 2013 octubre. Disponible en: URL: http:// www.minambiente.gov.co/images/BosquesBiodiversidadyServiciosEcosistemicos/pdf/nodos territoriales/250314 inf linea base adaptacion_cambio clima.pdf. Consultado: 6-08-2017.

ACEVEDO, A. 2016. Materiales educativos sobre uso y conservación del bosque seco tropical en el Caribe colombiano: una guía para facilitadores. Tropenbos Internacional Colombia \& Fondo Patrimonio Natural. Bogotá, Colombia.109:41-42.

ARNERO, M. 2015. La vaina del mezquite (Prosopis spp) en la alimentación del ganado. Trabajo presentado como requisito para optar título de Médico Veterinario Zootecnista. Universidad Autónoma Agraria Antonio Narro. Torreón, México.

BARBOSA, C.; RUÍZ, C.; GARCÍA, H.; GUTIÉRREZ, T.; RODRÍGUEZ, J.; MÁRQUEZ, W. 2008. Guía ilustrada de plantas destacadas del Santuario de Vida Silvestre Los Besotes. Panamericana, Formas e Impresos, S.A. Bogotá, Colombia. 
BARBOSA, O. 2013. Calentamiento Global: La máxima Expresión de la Civilización Petrofósil. Revista del CESLA. 16:35-68.

CAMPO, A. 2014. Diversidad y valor de importancia para la conservación de la vegetación natural. Parque Nacional Lihué Calel (Argentina). Anales de Geografía 34(2):25-42.

CEPAL. 2013. Panorama del cambio climático en Colombia. Colombia. 106:(9):15-20.

$\mathrm{CHUNCHO}$, C. 2011. Análisis de la percepción y medidas de adaptación al cambio climático que implementan en la época seca los productores de leche en Río Blanco y Paiwas, Nicaragua. Tesis como requisito para optar grado de Magister Scientiae en Agricultura Ecológica. Centro Agronómico Tropical de Investigación y Enseñanza, Escuela de Posgrado. Turrialba, Costa Rica.

DELGADO, D.; HERRERA, R.; CAIRO, J.; ORTA, Y. 2014. Samanea saman, árbol multipropósito con potencialidades como alimento alternativo para animales de interés productivo. Revista Cubana de Ciencia Agrícola 48(3):205-212.

DEPARTAMENTO NACIONAL DE PLANEACIÓN (2015). Plan Nacional de Adaptación al Cambio Climático. Disponible en:

https://colaboracion.dnp.gov.co/CDT/DNP/PNACC_ABC $\% 20$ Adaptaci\%C3\%B3n\%20Bases $\% 20$ Conceptuales.pdf. Consultado: 10-06-2017.

FARFÁN, F. 2014. Agroforestería y Sistemas Agroforestales con Café. Blanecolor S.A.S. Manizales, Colombia. 342:19-28.

FEDERACIÓN NACIONAL DE GANADEROS. 2015. Muerte masiva de bovinos en Cesar a causa del verano. 2015 febrero. Disponible en: URL: http://www.fedegan.org.co/noticias/muerte-masiva-de-bovinos-en-cesar-causa-delverano. Consultado: 22-06-2017.

FORERO, E.; ROMERO, C. 2005. Estudios en Leguminosas Colombianas. Academia Colombiana de Ciencias Exactas, Físicas y Naturales. Colección Jorge Álvarez Lleras, № 25. Bogotá, Colombia. 174:11-12.

HURTADO, G. 2016. Análisis del comportamiento promedio y tendencias de largo plazo de la temperatura máxima media para las regiones hidroclimáticas de Colombia. IDEAM. 2016 enero-abril. Disponible en: URL: http://www. ideam.gov.co/documents/21021/21138/Analisis+del+Comportamiento+de+la+Temperatura+Maxima.pdf/2a2f247cf457-45f3-ac9a-f2a481f8daa6. Consultado: 17-04-2017.

GUTIÉRREZ, A. 2016. Pensar el pasado para construir el futuro. Memoria local y poblamiento del corregimiento de Guacochito, Valledupar. Observatorio de Territorios Étnicos y Campesinos. 2016 febrero- abril. Disponible en: URL:http://www.etnoterritorios.org/index.shtml?apc=c-xx-1-\&x=1056. Consultado: 23-06-2017.

HERRERA, J.; RESTREPO, S.; RAMOS, P.; ARANGO, L.; GUERRERO, C.; MÁRQUEZ, J.; QUIROZ, A.; ROMERO, R.; GUTIÉRREZ, A.; DELUQUEZ, M. 2017. Reglamento Interno del Consejo comunitario de "Arcilla, Cardón y Tuna". Corregimiento de Guacochito Cesar, Valledupar - Cesar. Facultad de Estudios Ambientales y Rurales, Departamento de Desarrollo Rural y Regional de la Universidad Javeriana. Bogotá, Colombia.

IDEAM, PNUD, MADS, DNP, CANCILLERÍA. 2011. Nuevos Escenarios de Cambio Climático para Colombia 2011-2100, Herramientas Científicas para la Toma de Decisiones - Enfoque Nacional - Departamental: Tercera Comunicación Nacional de Cambio Climático. 2015 marzo. Disponible en: URL: http://documentacion.ideam.gov. co/openbiblio/bvirtual/022964/documento_nacional_departamental.pdf. Consultado: 8-06-2017.

LEYVA, T. 2011. Metodología para la identificación y determinación de geoformas y sus principales variaciones temporales mediante el uso de sensores remotos. Caso Servita. [Tesis Magister en Ciencias Geología]. Universidad Nacional de Colombia, Facultad de Ciencias, Departamento de Geociencias, Programa de Maestría en Ciencias - Geología. Bogotá, Colombia.

OROZA, S. 2012. ¿Hablamos de cambio climático? Fundación BBVA. Ibersaf Industrial, S.A. España. 106:15-16. 
PAREDES, M. 2013. Fijación biológica de nitrógeno en leguminosas y gramíneas [en línea]. Trabajo Final de Ingeniería en Producción Agropecuaria. Facultad de Ciencias Agrarias. Universidad Católica Argentina.

PIZANO, C.; GARCÍA, H. 2014. El bosque seco Tropical en Colombia. Instituto de Investigación de Recursos Biológicos Alexander Von Humboldt (IAvH). Bogotá, Colombia.

PLAN DE DESARROLLO DE VALLEDUPAR. 2012. Hacia la Transformación de Valledupar. 2012-2015 marzo. Disponible en: URL: http://carbonn.org/uploads/tx_carbonndata/Plan\%20Desarrollo\%20Valledupar\%202012\%20 -\%202015.pdf. Consultado: 15-10-2016.

PLAN DE DESARROLLO DEL DEPARTAMENTO DEL CESAR. 2012. Prosperidad a Salvo. Gobernación del Cesar. 2012-2015 marzo. Disponible en: URL: http://cesar.gov.co/d/index.php/es/menvertpolpla/menvertplandes. Consultado: 15-10-2016.

SARMIENTO, T. 2012. Impacto del procesamiento sobre la pared celular y las propiedades hipoglucémicas y tecnofuncionales de leguminosas. [Tesis doctoral]. Universidad Autónoma de Madrid. Madrid, España.

SOLANO, H. 2013. El algarrobo o samán, excelente alternativa ganadera. Ecología con Asocebú. 2013 abril. Disponible en: URL: http://ecologiasocebu.blogspot.com.co/2013/04/el-algarrobo-o-saman-excelente.html. Consultado: 10-04-2017.

STAPLES, G.; ELEVITCH, C. 2006. Samanea samam (rain tree). Species Profles for Pacifc Island Agroforestry. 2006. Disponible en: URL: https://raskisimani.files.wordpress.com/2013/01/samanea-raintree.pdf. Consultado: 12-02-2018.

TAPASCO, J.; MARTÍNEZ, S.; CALDERÓN, G.; ROMERO, D.; ORDÓÑEZ, A.; ÁLVAREZ, L.; SÁNCHEZ-ARAGÓN; LUDEÑA, C. 2015. Impactos Económicos del Cambio Climático en Colombia: Sector Ganadero. Banco Interamericano de Desarrollo, Monografía No. 254. 236: 5, 8,16.

VILLAREAL, H.; ÁlVAREZ, M.; CÓRDOBA, S.; ESCOBAR, F.; FAGUA, G.; GAST, F.; MENDOZA, H.; OSPINA, M.; UMAÑA, A. 2014. Manual de métodos para el desarrollo de inventarios de biodiversidad. Programas de inventarios de biodiversidad. Instituto de Investigación de Recursos Biológicos Alexander Von Humboldt. Bogotá, Colombia. 